\title{
ESTRATEGIAS DE AFRONTAMIENTO COGNITIVO, AUTO-EFICACIA Y VARIABLES LABORALES. ORIENTACIONES PARA PREVENIR EL ESTRÉS DOCENTE \\ COGNITIVE COPING SKILLS, SELF-EFFICACY AND WORK VARIABLES. GUIDELINES FOR PREVENTING STRESS OF TEACHERS
}

\author{
M. Elena Gismero-GonZÁLEZ \\ LAURA BERMEJO \\ María Prieto \\ Virginia CAgigal \\ Ana García Mina \\ ViCENTE HERNÁNDEZ
}

Departamento de Psicología. Facultad de Ciencias Humanas y Sociales. Universidad Pontificia Comillas egiesmero@chs.upcomillas.es

Cómo referenciar este artículo/How to reference this article:

Gismero-González, M. E., Bermejo, L., Prieto, M., Cagigal, V., García Mina, A., y Hernández, V. (2012). Estrategias de Afrontamiento Cognitivo, Auto-eficacia y variables laborales. Orientaciones para prevenir el Estrés Docente. Acción Psicológica, 9(2), 87-96 doi: http://dx.doi.org/10.5944/ap.9.2.4107

\section{Resumen}

Los profesores se enfrentan a menudo a muy diversas situaciones de estrés en su trabajo que pueden llegar a poner en juego su bienestar psicológico. Las denominadas estrategias de afrontamiento son respuestas ante una situación estresante puestas en marcha para tratar de manejarla o neutralizarla. Se analiza la relación de las estrategias de afrontamiento cognitivo con auto-eficacia y con variables laborales en una muestra de 413 profesores. La evaluación se realizó mediante un cuestionario donde se presentaron tres situaciones estresantes diferentes, junto a ítems del Brief COPE, del CERQ, y otros ítems diseñados para evaluar el afrontamiento cognitivo. La auto-eficacia se evaluó preguntando a los profesores en qué medida se percibían capaces de afrontar cada situación de estrés con éxito. Como variables laborales se

Agradecimientos: Este trabajo ha sido financiado por el Ministerio de Educación y Ciencia SEJ2007-60465/ EDUC. 
evaluaron: titularidad del centro, nivel de enseñanza y utilidad de la formación recibida para afrontar ese tipo de situaciones. Los resultados indican que los profesores de centros públicos utilizan más las estrategias de Pasividad pesimista y Autorreferencia Obsesiva que los de centros privados. Los de Infantil y Primaria emplean más la Planificación Racional ante los problemas de conducta de los alumnos y menos la Pasividad Pesimista ante la sobrecarga de trabajo que los de Secundaria. También aparecen diferencias significativas en Pasividad Pesimista y Planificación racional en función del nivel de percepción de eficacia. Finalmente, quienes encuentran más útil la formación recibida usan menos estrategias como Pasividad Pesimista y Autorreferencia Obsesiva. Estos resultados sugieren la importancia de adecuar las intervenciones para prevenir el estrés al nivel educativo y tipo de centro en que trabajen los docentes, así como la necesidad de diseñar iniciativas formativas de calidad que resulten útiles para su práctica cotidiana y les hagan percibirse significativamente más eficaces.

Palabras clave: estrategias de afrontamiento cognitivo, auto-eficacia, variables laborales, estrés docente.

\section{Abstract}

Teachers often face a variety of stressful situations at their work that may come to threaten their psychological well being. The so-called coping strategies are responses to a stressful situation implemented to try to manage or neutralize it. We analyze the relationship between cognitive coping strategies, self-efficacy, and job variables in a sample composed by 413 teachers. The evaluation was conducted through a questionnaire with 3 different stressful situations, as well as items of the Brief COPE, the CERQ, and other items designed to assess cognitive coping. Self-efficacy was evaluated asking the teachers to what extent they feel able to cope with each situation of stress successfully. The job variables evaluated were: type of school, educational level, and usefulness of received training to face this kind of situations. The results show that teachers of public schools use more the strategies of Pessimistic Passivity and Obsessive Self-Reference than those teachers of private schools. Teachers of Infant and Primary Education use more the Rational Planning when they face problems of students' behavior, and also use less Pessimistic Passivity when they are work overloaded, than teachers of Secondary Education. We also found significant differences in Pessimistic Passivity and Rational Planning according to the level of efficacy perceived. Finally, those who consider more useful their training use fewer strategies like Pessimistic Passivity and Obsessive Self-Reference. The results suggest the importance of adequate the interventions to prevent teachers stress to their educative level of teaching and to the kind of school in which their work. Also the results point out that it is necessary to design trainings of quality for teachers that became useful for their daily work and make them feel with much more efficacy.

Keywords: coping skills strategies, self-efficacy, job variables, and teachers stress.

\section{Introducción}

En las últimas décadas al estudio del estrés se ha unido el estudio de las estrategias puestas en marcha para hacerle frente y amortiguar sus efectos. Las denominadas «estrategias de afrontamiento» son respuestas ante una situación estresante puestas en marcha para tratar de manejarla o neutralizarla. Desde la teoría social cognitiva, las creencias de auto-eficacia juegan un papel modulador entre los estresores o las demandas del trabajo y la conducta de afrontamiento: las personas con un elevado nivel de auto-eficacia emplearán más estrategias de afrontamiento activo, centradas en la solución del problema, que las personas con niveles menores de auto-eficacia. Quienes tienen un alto nivel de auto-eficacia confían en su capacidad para manejar adecuadamente las demandas ambientales de su entorno, lo que hace que perciban las situaciones estresantes más como un reto que como una amenaza incontrolable (Bandura, 1997, 2001).

La auto-eficacia no sólo puede amortiguar las exigencias ambientales minimizando el im- 
pacto de sus efectos negativos, sino que incluso puede aportar bienestar cuando se responde de manera adecuada a los requerimientos ambientales (Salanova, Grau, Llorens y Schaufelli, 2001). La autoeficacia se ha asociado con la dedicación, la persistencia y la satisfacción en las acciones que realizamos (e.g., Salanova, Llorens, Cifre, Martínez y Schaufelli, 2003).

Los profesores se enfrentan a menudo a situaciones de estrés que pueden llegar a poner en juego su bienestar psicológico en el trabajo. En la literatura sobre estrés laboral de las últimas décadas hay numerosos estudios dirigidos a investigar este problema en este colectivo (e.g. Kyriacou, 2001; Travers y Cooper, 1997). Incluso en los medios de comunicación aparecen con frecuencia noticias relativas a la complejidad del trabajo de profesor actualmente y a los numerosos estresores a los que parece estar sometido el colectivo docente. Entre estos se han destacado las dificultades para hacer frente a los problemas de comportamiento y falta de motivación de los estudiantes, la diversidad del alumnado y los escasos recursos para afrontarla, la sobrecarga de funciones y tareas y la falta de tiempo para realizarlas. Estas demandas en el trabajo de los docentes junto con las continuas reformas en la legislación educativa, y los cambios en las familias y su relación con la escuela, parecen situar con frecuencia al profesor en situaciones de conflicto y ambigüedad en el desempeño de su rol. Entre las situaciones que los profesores identifican como más estresantes destacan principalmente: los problemas en el comportamiento de los alumnos (desde la falta de atención o participación en las tareas hasta conductas negativistas o incluso agresiones), la sobrecarga de trabajo y falta de tiempo para realizar distintas tareas y el conflicto o ambigüedad en el desempeño del rol de profesor (Hakanen, Bakker y Schaufeli, 2006; Manassero, García, Marques, Ferrer, Ramis y Gili, 2005; Marqués, Lima y Lopes, 2005; Salanova, Martínez y Lorente, 2005).

Por otro lado, los profesores encuentran escasos los recursos tanto personales como institucionales de que disponen para hacer frente a este tipo de situaciones estresantes en su trabajo. Manifiestan además haber recibido escasa o inadecuada formación, tanto inicial como continua, en este sentido (Marqués, Lima y Lopes, 2005; Van der Doef y Maes, 2002).

El fenómeno del estrés docente parece estar relacionado con serias consecuencias como la falta de implicación, absentismo, menor rendimiento, bajas médicas, elevado gasto en sustituciones y abandono de la profesión (Tang, Au, Schwarzer y Schmitz, 2001), siendo identificado el estrés como una de las causas de dicho abandono (Cifre, Llorens, Salanova y Martínez, 2003; Moriana y Herruzo, 2004). Asimismo, diversas investigaciones han coincidido en afirmar que el estrés docente, cuando se convierte en crónico, puede derivar en síndrome de burnout (e.g. Gil Monte, Peiró y Valcárcel, 1995; Guerrero, 1998). Por ello, este trabajo pretende analizar cuáles son las estrategias de afrontamiento empleadas por los profesores para hacer frente a situaciones de estrés como las descritas anteriormente. Concretamente nos centraremos en estudiar las estrategias de carácter cognitivo, escasamente consideradas en la investigación previa, en relación con la expectativa de eficacia que tienen los profesores respecto al afrontamiento de diversas situaciones estresantes, así como con distintas variables: titularidad del centro, nivel de enseñanza, auto-eficacia percibida y utilidad de la formación recibida. Para ello, previamente se realizará un estudio de la fiabilidad y validez factorial de la Escala de Recursos Personales de Afrontamiento Cognitivo, ya que en ella se incluyen algunos ítems de nueva creación, dada la escasez de instrumentos que evalúan con detalle esta variable.

\section{Método}

\section{Participantes}

Para realizar este estudio se ha analizado una muestra incidental de carácter no probabilístico formada por 413 profesores, de los cuales el $28.4 \%$ eran hombres y el $71.6 \%$ mujeres. La gran mayoría $(84.9 \%)$ procedían de la Comunidad de Madrid.

La media de edad de la muestra era 40.5 años (SD = 10.8), con una edad mínima de 21 años y una edad máxima de 67 años. El pro- 
medio de experiencia docente de los participantes era de 15.08 años $(S D=11.5)$. El 72.5\% de la muestra trabajaba en colegios privados concertados, el $2.2 \%$ en colegios privados y el $25.3 \%$ en centros públicos.

Respecto al nivel de enseñanza en el que trabajan los participantes, el mayor porcentaje se dedicaba a Educación Primaria (37.3\%), o a E.S.O. (29\%), $14.1 \%$ a Educación Infantil, 10\% a Bachillerato, $2 \%$ a F.P. y el $7.6 \%$ restante a «otros» (por ejemplo, Educación Especial).

\section{Instrumentos}

Escala de Recursos Personales de Afrontamiento Cognitivo. La evaluación se realizó mediante un cuestionario donde se presentaron tres situaciones estresantes diferentes (problemas de conducta de los alumnos; sobrecarga de trabajo y falta de tiempo; conflicto y ambigüedad de rol), ante las que se les planteaba la pregunta «¿Qué piensa usted al encontrarse en esta situación?». A continuación se presentaban 27 afirmaciones sobre las que tenían que responder en qué medida pasan por su cabeza dichos pensamientos, con una escala de respuesta que va desde $1=$ Nada a $8=$ Mucho. Algunos de los ítems se seleccionaron del Brief COPE (Carver, 1997), algunos ítems del Cognitive Emotion Regulation Questionnaire (CERQ) (Garnesfky, Kraaij y Spinhoven, 2002) y otros ítems diseñados para evaluar el afrontamiento cognitivo. Los ítems del Brief COPE y del CERQ se seleccionaron en base a sus pesos factoriales y a su validez teórica. El motivo de crear ítems nuevos fue la escasez de instrumentos que evalúan este tipo de afrontamiento con detalle, especialmente en lo relativo a distorsiones cognitivas que con frecuencia se activan en el pensamiento de las personas a la hora de afrontar situaciones difíciles. Ya que algunos ítems de esta escala son de creación propia se ha llevado a cabo un análisis más concreto de la validez factorial y la fiabilidad de este instrumento aplicado a cada una de las tres situaciones de estrés presentadas. Los resultados de estos análisis se presentan resumidamente en el apartado Resultados.

La Autoeficacia se evaluó preguntando a los profesores en qué medida se percibían capaces de afrontar con éxito cada una de las tres situaciones de estrés (con una escala de respuesta de 1 = Nada a $6=$ Mucho $)$.

Como variables laborales se evaluaron: titularidad del centro de trabajo (público, privado o privado-concertado), nivel de enseñanza en el que imparte la docencia (Educación Infantil, Primaria, ESO, Bachillerato, Formación Profesional u otros) y utilidad de la formación recibida para afrontar ese tipo de situaciones.

\section{Procedimiento}

Se contactó con diversos centros educativos mediante un correo electrónico enviado a los directores, dándoles a conocer la investigación y solicitando su colaboración. En algunos centros, un miembro del equipo investigador fue invitado a exponer brevemente al claustro los objetivos del estudio. A aquellos centros que decidían participar se les enviaba el número de cuestionarios que el centro solicitaba, cada uno con un sobre franqueado para su devolución por correo postal tras ser completado. Los profesores respondieron a los cuestionarios entre mayo de 2007 y mayo de 2009.

Todos los participantes de la investigación y la información obtenida fueron tratados de acuerdo con los principios éticos para la investigación científica. Tanto los procesos de obtención como de tratamiento y comunicación de los datos de esta investigación han sido adecuados a las directrices de la Ley Orgánica 15/99, de 13 de diciembre, de protección de datos de carácter personal (LOPDCP).

Los análisis estadísticos se realizaron mediante el programa SPSS 15.0.

\section{Diseño}

La metodología empleada es de naturaleza no experimental; se trata de un estudio ex-post facto. El diseño corresponde a una estrategia de tipo trans-seccional en la que empleamos dos cohortes con grupos de sujetos distintos (Maestros de Educación Infantil y Primaria y profesores de las etapas de ESO, Bachillerato o Formación Profesional). 


\section{Resultados}

El análisis factorial (por el método de extracción de componentes principales y de rotación varimax) de la escala de Afrontamiento Cognitivo arrojó una estructura de cuatro factores en cada una de las situaciones de estrés planteadas, excepto en la situación de problemas de conducta que presentaba un factor más denominado Escape Cognitivo. El total de varianza explicada por los 5 factores de la Situación 1 fue de 54.11\%; la varianza total explicada por los 4 factores de la Situación 2 fue de $55.15 \%$ y los 4 factores de la Situación 3 explicaron el $54.08 \%$ de la varianza total. En la Tabla 1 se presentan los porcentajes de varianza explicados por cada factor, así como la fiabilidad de cada uno de ellos, en las tres situaciones de estrés evaluadas.
Estos factores se conceptualizaron de la siguiente forma:

- Pasividad Pesimista: tendencia a centrarse y a anticipar los aspectos negativos de las situaciones estresantes, maximizando su importancia, atribuyendo sus causas a factores externos y resignándose a ellos.

- Autorreferencia Obsesiva: implica procesos de pensamiento centrados en uno mismo, auto-culpabilizadores, negativistas y de corte obsesivo y perfeccionista.

- Planificación Racional: recoge una serie de ítems que contemplan procesos de pensamiento centrados en la solución de problemas: definición del problema y de las metas a alcanzar, generar alternativas de solución y valoración de las mismas, planificación de la estrategia a llevar a cabo para solventarlo.

Tabla 1

Varianza explicada y fiabilidad de los factores en cada situación de estrés evaluada

\begin{tabular}{|c|c|c|c|c|c|}
\hline \multicolumn{6}{|c|}{ Situación 1: Problemas de conducta de los alumnos } \\
\hline & $\begin{array}{c}\text { Factor } 1 \\
\text { Pasividad } \\
\text { Pesimista }\end{array}$ & $\begin{array}{l}\text { Factor } 2 \\
\text { Autorref. } \\
\text { obsesiva }\end{array}$ & $\begin{array}{l}\text { Factor } 3 \\
\text { Planific. } \\
\text { racional }\end{array}$ & $\begin{array}{c}\text { Factor } 4 \\
\text { Reinterp. } \\
\text { positiva }\end{array}$ & $\begin{array}{c}\text { Factor } 5 \\
\text { Escape } \\
\text { cognitivo }\end{array}$ \\
\hline \% varianza explicada & 13.85 & 12.30 & 11.18 & 9.13 & 7.65 \\
\hline Alpha de Cronbach & .804 & .773 & .809 & .662 & .624 \\
\hline \multicolumn{6}{|c|}{ Situación 2: Sobrecarga de trabajo } \\
\hline & $\begin{array}{c}\text { Factor } 1 \\
\text { Pasividad } \\
\text { Pesimista }\end{array}$ & $\begin{array}{l}\text { Factor } 2 \\
\text { Planific. } \\
\text { racional }\end{array}$ & $\begin{array}{l}\text { Factor } 3 \\
\text { Autorref. } \\
\text { obsesiva }\end{array}$ & $\begin{array}{c}\text { Factor } 4 \\
\text { Reinterp. } \\
\text { positiva }\end{array}$ & \\
\hline$\%$ varianza explicada & 19.99 & 13.02 & 12.20 & 9.94 & \\
\hline Alpha de Cronbach & .87 & .791 & .705 & .642 & \\
\hline \multicolumn{6}{|c|}{ Situación 3: Conflicto y ambigüedad de rol } \\
\hline & $\begin{array}{c}\text { Factor } 1 \\
\text { Pasividad } \\
\text { Pesimista }\end{array}$ & $\begin{array}{l}\text { Factor } 2 \\
\text { Planific. } \\
\text { racional }\end{array}$ & $\begin{array}{l}\text { Factor } 3 \\
\text { Autorref. } \\
\text { obsesiva }\end{array}$ & $\begin{array}{c}\text { Factor } 4 \\
\text { Reinterp. } \\
\text { positiva }\end{array}$ & \\
\hline$\%$ varianza explicada & 16.19 & 14.08 & 13.21 & 10.61 & \\
\hline Alpha de Cronbach & .841 & .857 & .731 & 678 & \\
\hline
\end{tabular}


- Reinterpretación Positiva: los ítems que lo configuran se refieren a pensamientos positivos que tratan de reconvertir una situación difícil o generadora de estrés en algo mucho menos grave o preocupante y de lo que se puede extraer algún aspecto positivo o beneficioso.

- Escape Cognitivo: los ítems que lo forman se refieren a intentos de evasión cognitiva de la situación de estrés.

A continuación, presentamos las diferencias de medias que resultaron significativas en los factores de Afrontamiento Cognitivo en cada una de las tres situaciones en función de las variables estudiadas:

\section{A. Titularidad del centro:}

En función de la titularidad del centro en el que trabajan los profesores, se encontró que los que trabajan en centros públicos tuvieron medias más altas en Pasividad Pesimista y en Autorreferencia Obsesiva ante los Problemas de conducta de los alumnos que quienes trabajan en centros privados, siendo moderadas las magnitudes de estas diferencias $(\mathrm{d}=.46 \mathrm{y} \mathrm{d}=.41$, respectivamente). Los datos se recogen la Tabla 2.

Se encontraron también diferencias estadísticamente significativas en la estrategia $A u$ - torreferencia Obsesiva en la situación de Sobrecarga de trabajo en función de la Titularidad del centro $(\mathrm{t}=2.39, p=.02, \mathrm{~d}=.27)$; aquellos profesores que trabajaban en centros públicos empleaban en mayor medida esta estrategia.

Por último, ante la situación de Conflicto y ambigüedad de rol, también encontramos una diferencia significativa el uso de la estrategia Pasividad Pesimista en función de la titularidad del centro de trabajo: los profesores que desempeñaban su tarea en centros públicos empleaban en mayor medida esta estrategia $(\mathrm{t}=2.01, p=.45, \mathrm{~d}=.23)$. No obstante, esta diferencia resultó ser pequeña.

\section{B. Nivel de enseñanza:}

Con respecto al nivel de enseñanza impartido, encontramos dos diferencias significativas entre los profesores de enseñanza Infantil y Primaria vs. los de Secundaria: 1) en la situación Problemas de conducta de los alumnos, los profesores de infantil y primaria presentaron una media superior en el uso de la Planificación Racional ( $\mathrm{t}=3.18, p=.01, \mathrm{~d}=.33)$, y 2$)$ en la situación de Sobrecarga laboral, los profesores de Educación secundaria utilizan más la Pasividad Pesimista que los de infantil o primaria $(\mathrm{t}=2.13, p=.03)$, aunque la diferencia es pequeña $(\mathrm{d}=.23)$.

Tabla 2

Diferencias en Pasividad Pesimista y en Autorreferencia Obsesiva en Situación 1 (Problemas de conducta) en función de variables laborales (Prueba T)

\begin{tabular}{|c|c|c|c|c|c|c|c|c|c|}
\hline \multirow{2}{*}{ Factor } & \multicolumn{5}{|c|}{$\begin{array}{l}\text { Variables en las que aparecen diferencias } \\
\text { significativas y estadísticos descriptivos }\end{array}$} & \multirow{2}{*}{$\mathbf{T}$} & \multirow{2}{*}{ GI } & \multirow{2}{*}{$\begin{array}{c}\text { Sig. } \\
\text { (bilateral) }\end{array}$} & \multirow{2}{*}{$\begin{array}{l}\text { d de } \\
\text { Cohen }\end{array}$} \\
\hline & Variable & Valores & $\mathbf{N}$ & Media & DT & & & & \\
\hline \multirow{2}{*}{$\begin{array}{l}\text { F1. Pasividad Pesimista } \\
\text { ante problemas de } \\
\text { conducta }\end{array}$} & \multirow{2}{*}{$\begin{array}{l}\text { Titularidad } \\
\text { del centro }\end{array}$} & Público & 101 & 20.22 & 7.613 & \multirow{2}{*}{3.823} & \multirow{2}{*}{147.25} & \multirow{2}{*}{.000} & \multirow{2}{*}{0.46} \\
\hline & & Privado & 291 & 17.01 & 6.114 & & & & \\
\hline \multirow{2}{*}{$\begin{array}{l}\text { F 2. Autorreferencia } \\
\text { Obsesiva }\end{array}$} & \multirow{2}{*}{$\begin{array}{l}\text { Titularidad } \\
\text { del centro }\end{array}$} & Público & 100 & 15.99 & 6.088 & \multirow{2}{*}{3.344} & \multirow{2}{*}{143.30} & \multirow{2}{*}{.001} & \multirow{2}{*}{0.41} \\
\hline & & Privado & 290 & 13.75 & 4.778 & & & & \\
\hline
\end{tabular}




\section{Autoeficacia:}

En esta variable se dividió inicialmente a los sujetos en 3 grupos, en cada una de las 3 situaciones de estrés planteadas: a) Bajas expectativas de afrontar con éxito la situación: los que puntuaron 1 o 2; b) Expectativas medias de afrontar con éxito la situación: los que puntuaban 3 o 4, y c) Altas expectativas de afrontar la situación con éxito: los que puntuaban 5 o 6. Dado que el tamaño del grupo Bajas expectativas era muy pequeño en las 3 situaciones (Sit. $1 \mathrm{n}=8$; Sit. $2 \mathrm{n}=7$; Sit. 3 $\mathrm{n}=5$ ), las comparaciones se realizaron solamente entre los grupos de expectativas medias y altas.

Como puede observarse en las Tablas 3, 4 y 5 hay varias diferencias significativas en función de las expectativas de los profesores de afrontar con éxito las diferentes situaciones de estrés (Valoración secundaria). Los que tienen mayores expectativas de afrontar con éxito los problemas de conducta de los alumnos emplean menos la Pasividad pesimista, y el Escape cognitivo, y en cambio utilizan más la Planificación racional en esta situación. Ante la situación de Sobrecarga, los profesores con más expectativas de superarla con éxito obtienen significativamente mayores puntuaciones en Planificación racional y en reinterpretación positiva.

Por último, aquellos profesores que consideraron menores sus posibilidades de afrontar con éxito la situación de Conflicto y ambigüedad de rol obtuvieron una media más baja en Planificación racional que los que tenían mayores expectativas en esta situación $(\mathrm{t}=2.77$, $p=.01, \mathrm{~d}=.47)$.

Tabla 3

Diferencias en Pasividad Pesimista, Planificación Racional y Escape Cognitivo en Situación 1 (Problemas de conducta) en función de la Autoeficacia (Prueba T)

\begin{tabular}{|c|c|c|c|c|c|c|c|c|c|}
\hline \multirow{2}{*}{ Factor } & \multicolumn{5}{|c|}{$\begin{array}{c}\text { Variables en las que aparecen diferencias significativas } \\
\text { y estadísticos descriptivos }\end{array}$} & \multirow{2}{*}{$\mathbf{T}$} & \multirow{2}{*}{ GI } & \multirow{2}{*}{$\begin{array}{c}\text { Sig. } \\
\text { (bilateral) }\end{array}$} & \multirow{2}{*}{$\begin{array}{l}\text { d de } \\
\text { Cohen }\end{array}$} \\
\hline & Variable & Valores & $\mathbf{N}$ & Media & DT & & & & \\
\hline \multirow{3}{*}{$\begin{array}{l}\text { F1. Pasividad } \\
\text { Pesimista ante } \\
\text { problemas de } \\
\text { conducta }\end{array}$} & \multirow{7}{*}{$\begin{array}{l}\text { Evaluación } \\
\text { Secundaria } \\
\text { en Situación } 1\end{array}$} & Baja expectativa de éxito & 8 & 25.88 & 10.1 & 2.814 & 7.23 & .025 & 1.23 \\
\hline & & & & & & & & & \\
\hline & & Elevada expectativa de éxito & 163 & 15.77 & 5.89 & & & & \\
\hline \multirow{2}{*}{$\begin{array}{l}\text { F3. Planifica- } \\
\text { ción racional } \\
\text { ante problemas } \\
\text { de conducta }\end{array}$} & & Baja expectativa de éxito & 8 & 16.75 & 2.87 & -2.378 & 170 & .019 & 0.89 \\
\hline & & Elevada expectativa de éxito & 164 & 19.42 & 3.11 & & & & \\
\hline \multirow{2}{*}{$\begin{array}{l}\text { F5. Escape } \\
\text { Cognitivo ante } \\
\text { problemas de } \\
\text { conducta }\end{array}$} & & Baja expectativa de éxito & 8 & 9.13 & 3.72 & 3.308 & 170 & .001 & 1.00 \\
\hline & & Elevada expectativa de éxito & 164 & 5.92 & 2.62 & & & & \\
\hline
\end{tabular}


Tabla 4

Diferencias en Planificación Racional y en Reinterpretación Positiva en Situación 2 (Sobrecarga) en función de la Autoeficacia (Prueba T)

\begin{tabular}{|c|c|c|c|c|c|c|c|c|c|}
\hline \multirow{2}{*}{ Factor } & \multicolumn{5}{|c|}{$\begin{array}{c}\text { Variables en las que aparecen diferencias significativas } \\
\text { y estadísticos descriptivos }\end{array}$} & & \multirow{2}{*}{ GI } & \multirow{2}{*}{$\begin{array}{l}\text { Sig. } \\
\text { (bila- } \\
\text { teral) }\end{array}$} & \multirow{2}{*}{$\begin{array}{l}\text { d de } \\
\text { Cohen }\end{array}$} \\
\hline & Variable & Valores & $\mathbf{N}$ & Media & DT & & & & \\
\hline \multirow{2}{*}{$\begin{array}{l}\text { F2. } \\
\text { Planificación } \\
\text { Racional ante } \\
\text { sobrecarga }\end{array}$} & $\begin{array}{l}\text { Evaluación } \\
\text { Secundaria en }\end{array}$ & Baja expectativa de éxito & 7 & 15.43 & 2.149 & \multirow[t]{2}{*}{-2.649} & \multirow[t]{2}{*}{264} & \multirow[t]{2}{*}{.009} & \multirow[t]{2}{*}{1.21} \\
\hline & Situación 2 & Elevada expectativa de éxito & 259 & 18.91 & 3.457 & & & & \\
\hline \multirow{2}{*}{$\begin{array}{l}\text { F4. } \\
\text { Reinterpretac. } \\
\text { positiva ante } \\
\text { sobrecarga }\end{array}$} & & Baja expectativa de éxito & 7 & 15.14 & 6.04 & \multirow[t]{2}{*}{-3.388} & \multirow[t]{2}{*}{259} & \multirow[t]{2}{*}{.001} & \multirow[t]{2}{*}{-1.06} \\
\hline & & Elevada expectativa de éxito & 254 & 20.64 & 4.18 & & & & \\
\hline
\end{tabular}

Tabla 5

Diferencias en Pasividad Pesimista y en Planificación Racional en Situación 3 (Conflicto y Ambigüedad de rol) en función de la Autoeficacia (Prueba T)

\begin{tabular}{|c|c|c|c|c|c|c|c|c|c|}
\hline \multirow{2}{*}{ Factor } & \multicolumn{5}{|c|}{$\begin{array}{c}\text { Variables en las que aparecen diferencias significativas } \\
\text { y estadísticos descriptivos }\end{array}$} & \multirow{2}{*}{$\mathrm{T}$} & \multirow{2}{*}{ GI } & \multirow{2}{*}{$\begin{array}{l}\text { Sig. } \\
\text { (bila- } \\
\text { teral) }\end{array}$} & \multirow{2}{*}{$\begin{array}{l}\text { d de } \\
\text { Cohen }\end{array}$} \\
\hline & Variable & Valores & $\mathbf{N}$ & Media & DT & & & & \\
\hline \multirow{2}{*}{$\begin{array}{l}\text { F2. Planificación } \\
\text { Racional en } \\
\text { Conflicto y } \\
\text { Ambigüedad de rol }\end{array}$} & \multirow{2}{*}{$\begin{array}{l}\text { Evaluación } \\
\text { Secundaria en } \\
\text { Situación } 3\end{array}$} & Elevada expectativa de éxito & 194 & 16.14 & 6.708 & & & & \\
\hline & & Baja expectativa de éxito & 5 & 15.00 & 3.162 & -3.072 & 198 & .002 & 1.44 \\
\hline
\end{tabular}

D. Utilidad de la formación recibida:

Esta variable arroja diferencias significativas en las tres situaciones de estrés estudiadas:

1) Ante los problemas de conducta de los alumnos (Situación 1), los profesores que valoran como bastante o muy útil la formación que han recibido relacionada con la resolución de este tipo de problemas utilizan mucho menos la Pasividad pesimista $(\mathrm{t}=3.57, p<.001$, $\mathrm{d}=.61)$ y la Autorreferencia obsesiva $(\mathrm{t}=2.58, p=.01, \mathrm{~d}=.40)$;

2) Ante la sobrecarga laboral (Situación 2), los profesores que valoran bastante o muy útil su formación para manejar situaciones estresantes emplean en un grado mucho menor la Pasividad pesimista $(\mathrm{t}=2.95, p<.01, \mathrm{~d}=.49)$;
3) En la situación de Conflicto y ambigüedad de rol, los profesores que valoran como bastante o muy útil su formación para afrontar situaciones de estrés emplean mucho menos la Pasividad pesimista $(\mathrm{t}=2.77$, $p<.01, \mathrm{~d}=.47)$ y la Autorreferencia obsesi$v a(\mathrm{t}=3.32, p=.001, \mathrm{~d}=.56)$.

\section{Conclusiones}

Los resultados indican que los profesores de centros públicos utilizan en mayor medida estrategias de carácter reactivo (Pasividad pesimista y Autorreferencia Obsesiva) que los de centros privados. En relación con estos resultados no hemos encontrado estudios previos similares, por lo que sería interesante estudiar con más profundidad en el futuro estas diferencias en función de la Titularidad del centro docente. 
Los profesores de Infantil y Primaria emplean más la Planificación Racional frente a los problemas de conducta de los alumnos y menos la Pasividad Pesimista ante la sobrecarga de trabajo que los de Secundaria. En cuanto a la primera de estas diferencias, podría estar asociada con el tipo de problemática conductual de los alumnos, muy diferente en función del nivel en que se encuentren; quizá el profesor de infantil y primaria tiene en sus aulas alumnos que presenten problemas de conducta menos destacados, de modo que el docente disponga de más tiempo para planificar y emprender soluciones racionales meditadas, entre otras estrategias.

Los profesores con baja percepción de autoeficacia frente a los problemas de conducta de los alumnos utilizan más la Pasividad Pesimista y el Escape Cognitivo y menos la Planificación Racional que los que se perciben con elevada autoeficacia. Frente a la sobrecarga de trabajo, quienes se perciben con elevada auto-eficacia emplean en mayor medida la Planificación $R a$ cional y la Reinterpretación Positiva que los que se perciben poco auto-eficaces. Los profesores con baja percepción de eficacia emplean menos la Planificación Racional frente a situaciones de conflicto o ambigüedad de rol que los que tienen elevada percepción de eficacia. Así, los resultados de esta investigación muestran que la autoeficacia profesional se relaciona con el uso de distintas estrategias de afrontamiento ante diversas situaciones laborales estresantes: los profesores con mayores niveles de auto-eficacia utilizan significativamente más estrategias de afrontamiento eficaces, proactivas, mientras que aquellos con bajos niveles de auto-eficacia tienden a emplear ante las situaciones estresantes de su trabajo estrategias de afrontamiento menos eficaces, más evitativas. Estos resultados apoyan la teoría social cognitiva (Bandura, 1997, 2001) y están en la línea que indica que las personas con altos niveles de eficacia confían en sus habilidades y capacidad para responder a los estímulos del medio y pueden ejercer control sobre ellos. Coinciden también con los resultados encontrados por otros autores como Salanova, Grau y Martínez (2005), que hallaron en trabajadores de diferentes ocupaciones, entre ellos profesores, que aquellos con elevadas creencias de auto-eficacia mostraban más conductas de afrontamien- to activo o centrado en el problema que los que tenían menores niveles de auto-eficacia.

Por último, aquellos profesores que encontraron bastante o muy útil la formación recibida para afrontar situaciones de estrés usan en menor medida estrategias como la Pasividad Pesimista y la Autorrefencia Obsesiva. Por tanto, los resultados obtenidos señalan una clara vinculación entre la formación recibida, especialmente la que los profesores valoran como útil, con el hecho de emplear en mayor o menor medida estrategias más pasivas o reactivas, menos adecuadas y eficaces para afrontar con éxito las distintas demandas estresantes de su trabajo.

Estos resultados informan de la importancia de adecuar las intervenciones para prevenir el estrés al nivel educativo y tipo de centro en el que se encuentren los docentes, así como de la necesidad de diseñar iniciativas de formación de calidad que resulten de utilidad para su práctica cotidiana, les hagan percibirse significativamente más eficaces y emplear estrategias de carácter más proactivo, como la Planificación Racional, frente a otras más reactivas y pasivas, como la Pasividad Pesimista.

Los problemas de conducta de los alumnos, la sobrecarga laboral y el conflicto de rol laboral están presentes en los centros escolares, y probablemente sean inevitables. Cuando las situaciones generadoras de estrés son difíciles de controlar por parte del centro, una estrategia de prevención adecuada podría ser la mejora de la auto-eficacia profesional de los docentes, por ejemplo con programas de formación en los que se potencie la capacidad de control. Nuestro estudio sugiere que elevados niveles de auto-eficacia pueden ayudar a los profesores a afrontar de manera más efectiva los estresores (incluso que no sean percibidos como tales). Si la auto-eficacia se acompaña de uso de estrategias más activas y adecuadas para afrontar las demandas laborales, que amortiguan el impacto negativo de los estresores, su potenciación en el ámbito docente se plantea como un reto a conseguir. Tanto el incremento de la auto-eficacia como el desarrollo y puesta en práctica de estrategias de afrontamiento adecuadas frente a las demandas del trabajo contribuirán no sólo a reducir el estrés docente, sino a fomentar un mayor bienestar y satisfacción laboral entre los profesores. 


\section{Referencias}

Bandura, A. (1997). Self-efficacy: the exercise of control. New York, N.Y.: Freeman.

Bandura, A. (2001). Social cognitive theory: an agentic perspective. Annual Review of Psychology, 52, 1-26. doi: 10.1146/annurev.psych.52.1.1.

Carver, C. S. (1997). You Want to Measure Coping But Your Protocol's Too Long: Consider the Brief COPE. International Journal of Behavioral Medicine, 4(1), 92-100. doi: 10.1207/s15327558ijbm0401_6.

Cifre, E., Llorens, S., Salanova, M., y Martínez, I. (2003). Salud psicosocial en profesores: repercusiones para la mejora en la gestión de recursos humanos. [Psychosocial health in teachers: implications for improving human resources management]. Estudios financieros, Revista de Trabajo y seguridad Social, 247, 153-168.

Garnesfky, N., Kraaij, V., y Spinhoven, P. (2002). CERQ: Manual for the use of the Cognitive Emotion Regulation Questionnaire. Leiderdorp, Netherlands: DATEC.

Gil Monte, P. R., Peiró, J. M., y Valcárcel, P. (1995). A causal model of burnout process development: An alternative to Golembiewski and Leiter models. Actas del VII Congreso de Psicología del Trabajo y de las Organizaciones. Györ, Hungría.

Guerrero, E. (1998). "Burnout» o desgaste psíquico y afrontamiento del estrés en el profesorado universitario. [Burnout and stress coping in university faculty] (Tesis doctoral). Universidad de Extremadura, España.

Hakanen, J. J., Bakker, A. B., y Schaufeli, W. B. (2006). Burnout and engagement among teachers. Journal of School Psychology, 43, 495-513. http://dx.doi.org/10.1016/j.jsp.2005.11.001.

Kyriacou, C. (2001). Teacher stress: directions for future research. Educational Review, 53(1), 27-35. doi:10.1080/00131910120033628.

Manassero, M. A., García, E., Vázquez, A., Ferrer, V. A., Ramis, C., y Gili, M. (2000). Análisis causal del burnout en la enseñanza. [Causal analysis of burnout in teaching]. Revista de Psicología del Trabajo y de las Organizaciones, 16(2), 173-195.

Marques, A., Lima, M. L., y Lopes, A. (2005). Fuentes de estrés, burnout y estrategias de coping en profesores portugueses. [Sources of stress, burnout, and coping strategies in Portuguese teachers]. Revista de Psicología del Trabajo y de las Organizaciones, 21(1-2), 125-143.

Moriana, J. A., y Herruzo, J. (2004). Estrés y Burnout en profesores. [Stress and Burnout in teachers]. International Journal of Clinical and Health Psychology, 4(3), 597-621. Id: 40539.

Salanova, M., Grau, R., Llorens, S., y Schaufelli, W. B. (2001). Exposición a las tecnologías de la información, burnout y engagement: el rol modulador de la autoeficacia relacionada con la tecnología. [Exposure to information technology, burnout and engagement: the moderating role of self-efficacy related to technology]. Revista de Psicología Social Aplicada, 11, 69-90.

Salanova, M., Llorens, S., Cifre, E., Martínez, I. M., y Schaufelli, W. B. (2003). Perceived collective efficacy, subjective well-being and task performance among electronic work groups: an experimental study. Small Groups Resarch, 34(1), 43-73.

Salanova, M., Grau, R. M., y Martínez, I. M. (2005). Demandas laborales y conductas de afrontamiento: el rol modulador de la autoeficacia profesional. [Job demands and coping behaviour: the moderating role of professional self-efficacy]. Psicothema, 17(3), 390-395.

Salanova, M., Martínez, I. M., y Lorente, L. (2005). ¿Cómo se relacionan los obstáculos y facilitadores organizacionales con el burnout docente?: Un estudio longitudinal. [How do organizational obstacles and facilitators relate with teacher burnout? A longitudinal study]. Revista de Psicología del Trabajo y de las Organizaciones, 21(1-2), 37-54.

Tang, C., Au, W., Schwarzer, R., y Schmitz, G. (2001). Mental health outcomes of job stress among Chinese teachers: role of stress resource factors and Burnout. Journal of Organizational behavior, 22, 887-901. doi: 10.1002/job.120.

Travers, C. J., y Cooper, C. L. (1997). El estrés de los profesores. La presión de la actividad docente. [The stress of teachers. The pressure of teaching activity]. Barcelona, España: Paidós.

Van der Doef, M. y Maes, S. (2002). Teacher-specific quality of work versus general quality of work assessment: A comparison of the validity regarding burnout, (psycho)somatic well-being and job satisfaction. Anxiety, stress and coping, 15(4), 327-344. doi:10.1080/1061580021000056500. 